\title{
Both antenatal and postnatal inflammation contribute information about the risk of brain damage in extremely preterm newborns
}

\author{
Diana Yanni ${ }^{1}$, Steven J. Korzeniewski ${ }^{2}$, Elizabeth N. Allred ${ }^{3}$, Raina N. Fichorova ${ }^{4}$, T. Michael O'Shea $^{5}$, Karl Kuban ${ }^{6}$, \\ Olaf Dammann ${ }^{7}$, Alan Leviton ${ }^{3}$ for the ELGAN Study Investigators
}

BACKGROUND: Preterm newborns exposed to intrauterine inflammation are at an increased risk of neurodevelopmental disorders. We hypothesized that adverse outcomes are more strongly associated with a combination of antenatal and postnatal inflammation than with either of them alone.

METHODS: We defined antenatal inflammation as histologic inflammation in the placenta. We measured the concentrations of seven inflammation-related proteins in blood obtained on postnatal days 1, 7, and 14 from 763 infants born before 28 weeks of gestation. We defined postnatal inflammation as a protein concentration in the highest quartile on at least 2 days. We used logistic regression models to evaluate the contribution of antenatal and postnatal inflammation to the risk of neurodevelopmental disorders.

RESULTS: The risk of white matter damage was increased when placental inflammation was followed by sustained elevation of C-reactive protein or ICAM-1. We found the same for spastic cerebral palsy when placental inflammation was followed by elevation of TNF-a or IL-8. The presence of both placental inflammation and elevated levels of IL-6, TNF- $a$, or ICAM-1 was associated with an increased risk for microcephaly.

CONCLUSION: Compared with a single hit, two inflammatory hits are associated with stronger risk for abnormal cranial ultrasound, spastic cerebral palsy, and microcephaly at 2 years.

$E_{a}^{x}$ xtremely preterm-born infants are at an increased risk of a wide spectrum of neurodevelopmental impairment ranging from cognitive limitation and behavioral disorders to cerebral palsy $(1,2)$. The most common underlying pathology is cerebral white matter damage (3). The mechanisms leading to this white matter damage are complex, and a combination of developmental, cellular, and immunologic factors appear to be involved (4-6).
Inflammation as demonstrated by microglial activation and elevated levels of inflammatory mediators causes white matter damage during a developmentally vulnerable period (5). Various antenatal and postnatal stimuli can trigger perinatal inflammation. Preterm labor, preterm premature rupture of membranes, and chorioamnionitis are all associated with an increased risk of white matter damage and adverse neurodevelopmental outcome (7-11).

Postnatal inflammation-initiating illnesses such as bronchopulmonary dysplasia, necrotizing enterocolitis, and sepsis are risk factors for white matter damage and adverse neurodevelopmental outcome (12). Extremely preterm newborns who had elevated levels of biomarkers of systemic inflammation on two occasions 1 week apart are at a higher risk of ultrasound indicators of brain damage (13), severely low developmental indices (14), and microcephaly at 2 years (15) than newborns with no systemic inflammation.

Although antenatal and postnatal inflammation each alone can contribute to white matter damage, we wanted to see if the risk was higher when both inflammatory exposures were present. Our aim was to study a two-hit hypothesis for white matter damage, with antenatal inflammation being the first hit and persistent postnatal inflammation the second. The ELGAN Study of infants born before the 28th week of gestation collected data that allowed us to evaluate whether the co-occurrence of prenatal and postnatal indicators of inflammation was associated with higher risk of structural and functional indicators of cerebral white matter damage.

\section{METHODS}

The ELGAN study was designed to identify characteristics and exposures that increase the risk of structural and functional neurologic disorders in ELGANs (the acronym for Extremely Low Gestational Age Newborns, 16). During the years 2002-2004, women delivering before 28 weeks' gestation at one of the 14 participating institutions were asked to enroll in the study. Each institution's

\footnotetext{
${ }^{1}$ Division of Newborn Medicine, Department of Pediatrics, Floating Hospital for Children at Tufts Medical Center, Boston, Massachusetts; ${ }^{2}$ Department of Obstetrics and Gynecology, Wayne State University School of Medicine, Detroit, Michigan; ${ }^{3}$ Department of Neurology, Boston Children's Hospital and Harvard Medical School, Boston, Massachusetts; ${ }^{4}$ Departments of Obstetrics, Gynecology and Reproductive Biology, Brigham and Women's Hospital and Harvard Medical School, Boston Massachusetts; ${ }^{5}$ Department of Pediatrics, University of North Carolina, Chapel Hill North Carolina; ${ }^{6}$ Departments of Pediatrics and Neurology, Boston Medical Center and Boston University School of Medicine, Boston Massachusetts; ${ }^{7}$ Department of Public Health \& Community Medicine, Tufts University School of Medicine, Boston, Massachusetts. Correspondence: Diana Yanni (Dyanni@tuftsmedicalcenter.org) 


\section{Articles | Yanni et al.}

review board approved enrollment and consent procedures and documents.

The sample for this report consists of the 763 newborns for whom we had information about pathology and bacteriology of the placenta and protein concentrations in blood spots obtained on at least two of the three protocol days (days 1, 7, and 14), and who had a developmental assessment at age 2 years post-term equivalent. Those who had a motor handicap, which would interfere with assessments of cognition, were not included (i.e., inability to walk independently and Gross Motor Function Classification System level $>1$ ). The characteristics of the patient population can be found in Supplementary Table S1 online.

\section{Placenta}

Delivered placentas were placed in a sterile exam basin and transported to a sampling room, where they were biopsied under sterile conditions; $82 \%$ were obtained within 1 hour of delivery.

In keeping with the guidelines of the 1991 CAP Conference (17), representative sections were taken from all abnormal areas as well as routine sections of the umbilical cord and a membrane roll, and full thickness sections from the center and a paracentral zone of the placental disc. After training to minimize observer variability, study pathologists examined the slides for histologic characteristics $(18,19)$. For summary purposes, placental inflammation is defined as inflammation of the chorionic plate (grade 3, which is defined as neutrophils up to the amnionic epithelium and stage 3 , which is defined as $>20$ neutrophils/20 $\times$ ) or of the chorion/decidua (grade 3 , which required numerous large or confluent foci of neutrophils). Placental infection is defined by recovery of organisms regardless of the presence or absence of concomitant inflammation.

\section{Blood Spot Collection}

After blood was collected for clinical indications, drops were blotted on a filter paper on the first postnatal day (range: 1-3 days), the 7th postnatal day (range: 5-8 days), and the 14th postnatal day (range: 12-15 days). Dried blood spots were stored at $-70{ }^{\circ} \mathrm{C}$ in sealed bags with desiccant until they were processed. All references to protein concentrations refer to these three samples.

\section{Protein Measurement}

The Laboratory of Genital Tract Biology of the Department of Obstetrics, Gynecology and Reproductive Biology at Brigham and Women's Hospital, Boston measured 29 proteins with the Meso Scale Discovery (MSD) electrochemiluminescence system as previously described in detail elsewhere (43). Interassay variations are invariably $<20 \%$. Measurements of each protein were normalized to $\mathrm{mg}$ total protein. Levels of following proteins were evaluated in our study: (i) cytokines that initiate multiple inflammatory cascades (Interleukin (IL)-1beta, IL-6, and TNF-alpha (tumor necrosis factor-alpha)); (ii) a proinflammatory chemokine (IL-8 (CXCL8)) and adhesion protein (intercellular adhesion molecule-1(ICAM-1) (CD54)) that represent proteins upregulated by multiple inflammatory pathways initiated by IL-1beta, TNF-alpha, and IL-6, and released in the circulation during inflammation); and (iii) liver-produced acute reactive proteins that are upregulated early in the inflammatory process and those that persist during chronic systemic inflammation (CRP (C-reactive protein), SAA (serum amyloid A)). We focused on this limited set of inflammation-associated proteins because they were the most informative in previous analyses of white matter damage in the ELGAN sample $(13,14,15)$.

In previous analyses in this sample, protein elevations in the top quartile for gestational age and postnatal day on two separate days provided considerably more discriminating risk information than did elevations on just 1 day $(13,14,16)$. Thus, our indicator of postnatal systemic inflammation is a concentration of an inflammation-related protein in the top quartile that persisted or recurred, identified here as intermittent or sustained systemic inflammation (ISSI).

\section{Imaging/Cranial Ultrasonography}

Routine ultrasound examinations were performed by sonographers at each hospital using high-frequency transducers (7.5 and $10 \mathrm{MHz}$ ) between days 1 and 4, days 5 and 14, and between day 15 and the 40th week post menstruation. Several methods were employed to minimize observer variability. All ultrasound scans were read by two independent readers who did not have access to clinical information. A manual with definitions and instructions was distributed (20). Ventriculomegaly and hypoechoic lesion were the two indicators for white matter damage defined by cranial ultrasound. Cerebral white matter was divided into eight zones in each hemisphere; in each zone, lesions were characterized as hyperechoic and/or hypoechoic (i.e., pattern in echoes that is characteristically attributed to a presumed pathologic change in tissue density). Ventriculomegaly was defined visually using a template included on the data collection form. Additional details about obtaining and reading ultrasound scans are described elsewhere (21).

Because ventriculomegaly and a hypoechoic lesion are better predictors for cerebral palsy and low Bayley scores compared with intraventricular hemorrhage (20), we chose ventriculomegaly and a hypoechoic lesion as our cranial ultrasound outcomes.

\section{4-Month Assessment}

Overall, 91\% of surviving children returned for an assessment at around a 24 months' corrected age; $77 \%$ were evaluated between 23.5 and 27.9 months. Their assessment included measurement of head circumference, neurological exam, and developmental evaluation.

Certified examiners administered and scored the Bayley Scales of Infant Development- Second Edition (22). We chose as our main outcome a Mental and Psychomotor Development Index (MDI, PDI) $<55$, which is three SD below the expected mean, and therefore constitutes a severe impairment, and because the predictive ability of an MDI $<55$ is higher than that of a score below 70, which is two SD below the expected mean $(14,23)$. Because some test items require intact motor function when assessing mental development, we excluded children who were unable to walk independently (Gross Motor Function Classification System level >1).

\section{Data Analysis}

We evaluated the hypothesis that a combination of prenatal and postnatal inflammation together would be more strongly associated with white matter damage and neurodevelopmental disorders than with either of them alone. We defined the first hit, prenatal inflammation/infection as histologic inflammation/recovery of an organism in the placenta parenchyma. The second hit was postnatal inflammation defined by intermittent or recurrent elevations of seven inflammation-related protein concentrations. Logistic regression models were created to evaluate associations with indicators for white matter damage; multinomial models were developed for the evaluation of cerebral palsy subtypes. To calculate odds ratios and 95\% confidence intervals, we included three "dummy variables" for our first hit, second hit, and both hits together, as well as variables for gestational age categories (23-24, 25-26, and 27 weeks) and sex in our regression analyses. Head circumference at birth was also adjusted for the risk assessment of microcephaly. We did separate analyses with first hit being placental inflammation or placental infection. Indicators for white matter damage were ventriculomegaly or hypoechoic lesion on cranial ultrasound; neurodevelopmental disorders were cerebral palsy, Bayley scores $<55$, and microcephaly at 2 years' corrected age. When the $95 \%$ confidence intervals do not include 1.0 , the odds ratios are statistically significant.

\section{RESULTS}

Placental inflammation and postnatal systemic inflammation together were associated with a higher risk for all structural and clinical indicators of white matter damage except spastic hemiparesis (Table 1). Associations with ventriculomegaly, 
Table 1. Summary of results

\begin{tabular}{|c|c|c|c|c|c|c|c|c|c|c|c|c|c|c|}
\hline First hit: & \multicolumn{7}{|c|}{ Placental Inflammation } & \multicolumn{7}{|c|}{ Organisms in placenta } \\
\hline Second hit: & 造 & $\frac{n}{\frac{n}{u}}$ & 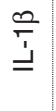 & $\stackrel{\varphi}{\perp}$ & 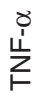 & $\stackrel{\stackrel{\infty}{\perp}}{=}$ & $\sum_{\underline{0}}^{\top}$ & के & $\frac{0}{\tilde{r}}$ & 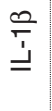 & $\stackrel{\varphi}{\stackrel{\varphi}{=}}$ & 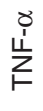 & $\stackrel{\stackrel{\infty}{\perp}}{=}$ & \\
\hline Outcomes: & & & & & & & & & & & & & & \\
\hline Ventriculomes & & & & & & & & & & & & & & \\
\hline Hypoechoic le & & & & & & & & & & & & & & \\
\hline Spastic quadr & & & & & & & & & & & & & & \\
\hline Spastic dipare & & & & & & & & & & & & & & \\
\hline $\mathrm{MDI}<55$ & & & & & & & & & & & & & & \\
\hline $\mathrm{PDI}<55$ & & & & & & & & & & & & & & \\
\hline Microcephaly & & & & & & & & & & & & & & \\
\hline
\end{tabular}

The first hit is placental inflammation or recovery of an organism from the placenta. The second hit is the elevated concentration of the cytokines in the top quartile for gestational and postnatal age. Outcome measures are ventriculomegaly, hypoechoic lesion, spastic quadriparesis, spastic diparesis, Mental Development Index <55, Psychomotor Development Index $<55$, and microcephaly (head circumference Z-score $<-2$ SD at 2 years' corrected age). Dark gray boxes indicate that when the first hit is followed by the second hit, there is a stronger association with the outcome than with either hit alone.

hypoechoic lesion, and microcephaly were more consistent with the two-hit hypothesis. Recovery of organisms from the placenta appeared to contribute to the risk of hypoechoic lesion and spastic diparesis. Among the cytokines, CRP, TNF- $\alpha$, IL-8, and ICAM-1 were most frequently observed in the associations supporting the two-hit hypothesis.

\section{Ventriculomegaly}

Placental inflammation in isolation was not associated with an increased risk of ventriculomegaly. Elevated levels of IL-1 $\beta$, IL-6, TNF- $\alpha$, and IL-8 on multiple days were associated with an increased risk of ventriculomegaly. However, among babies who had elevated levels of these cytokines, those who also had placental inflammation were not at an increased risk. When both placental inflammation and elevated levels of CRP and ICAM-1 occurred in succession, the risk for ventriculomegaly was increased. This was not the case when each of these factors were present alone (Figure 1 and Supplementary Figure S1).

Similarly, recovery of an organism from the placenta in isolation was not associated with an increased risk for ventriculomegaly. Elevated concentrations of TNF- $\alpha$, IL-8, and ICAM-1 on multiple days were associated with ventriculomegaly. When recovery of an organism from the placenta was followed by elevated levels of SAA, CRP, and IL-1 $\beta$, there was an increased risk for ventriculomegaly even though there was no risk associated with these factors alone.

\section{Hypoechoic lesion}

Placental inflammation when followed by recurrent or continued elevated concentrations of CRP or ICAM-1

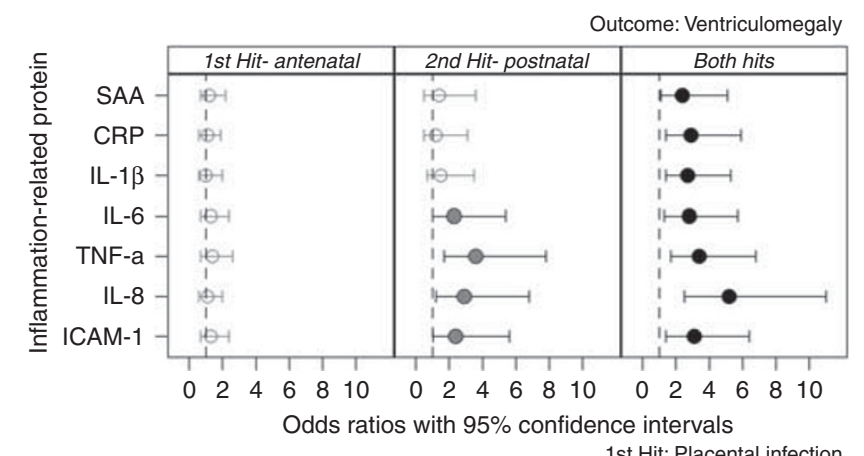

1st Hit: Placental infection
2nd Hit: Intermittent/sustained systemic inflammation

Figure 1. The odds ratio (OR) point estimates (circles) and confidence intervals (Cl) (length of the horizontal lines) for ventriculomegaly.

First column shows the risk for ventriculomegaly when there is only the first hit. Placenta infection is defined by the recovery of an organism from a biopsy of the placenta. The second column shows the risk for ventriculomegaly when there is only the second hit. The second hit, intermittent or sustained systemic inflammation, is defined by the levels of the cytokines seen on the left, in the top quartile for gestational and postnatal age. The third column shows the risk for ventriculomegaly when the first hit is followed by the second hit. OR and $95 \% \mathrm{Cl}$ for both hits are as follows: CRP $3(1.5,6.1)$, IL-1 $2.8(1.3,5.8)$, IL-6 2.6(1.2, 5.4), IL-8 $6.3(3,13)$, and ICAM-1 $3.1(1.5,6.5)$.

provided more information about the risk for a hypoechoic lesion than any of these factors alone (Figure 2 and Supplementary Figure S2).

Recovery of an organism from the placenta was associated with an increased risk of a hypoechoic lesion. Intermittent or sustained elevated concentrations of IL-8 only contributed to risk information about a hypoechoic lesion. When placental 


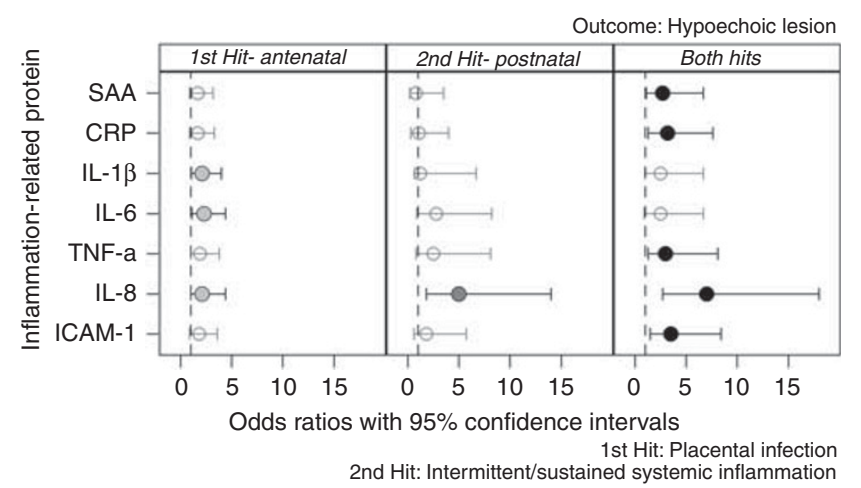

Figure 2. The odds ratio (OR) point estimates (circles) and confidence intervals $(\mathrm{Cl})$ (length of the horizontal lines) for hypoechoic lesion on cranial ultrasound. First column shows the risk for hypoechoic lesion when there is only the first hit, the recovery of an organism from the placenta. The second column shows the risk for hypoechoic lesion when there is only the second hit. The second hit is defined by the levels of the cytokines seen on the left, in the top quartile for gestational and postnatal age. The third column shows the risk for hypoechoic lesion when the first hit is followed by the second hit. OR and $95 \% \mathrm{Cl}$ for both hits are as follows: SAA $2.7(1.1,6.7)$, CRP $3.2(1.3,7.6)$, TNF-a $3(1.3,8.1)$, IL-8 $7(2.7,18)$, and ICAM-1 $3.5(1.5,8.4)$.

infection was followed by elevated levels of SAA, CRP, TNF- $\alpha$, IL-8, and ICAM-1, the risk for hypoechoic lesion was increased compared with that when these factors occurred alone.

\section{Spastic Diparesis}

The risk for spastic diparesis increased when placental inflammation was followed by elevated concentrations of TNF $\alpha$ or IL-8. Placental inflammation, especially with recovery of an organism, was associated with an increased risk for diparesis. When followed by elevated levels of CRP, IL-6, or ICAM-1, the risk was higher (Supplementary Figure S3a,b).

\section{Spastic Quadriparesis}

The two-hit hypothesis was supported when placental infection was followed by recurrent or sustained elevation of TNF- $\alpha$ or IL-8 (Supplementary Figure S4a,b).

\section{Spastic Hemiparesis}

Elevated levels of IL-6 were associated with an increased risk for hemiparesis. However, neither placental inflammation nor recovery of an organism from the placenta further increased the risk (Supplementary Figure S5a,b).

\section{Microcephaly}

The two-hit hypothesis was supported when placental inflammation with or without recovery of an organism was followed by elevated levels of IL- 6 , TNF- $\alpha$, or ICAM- 1 . When elevated concentration of IL-8 followed placental infection, the risk for microcephaly was increased compared with the risk provided by IL-8 alone (Supplementary Figure S6a,b).

\section{Low MDI}

The support for two separate inflammatory insults contributing to the pathogenesis of a very low MDI was demonstrated when placental inflammation was followed by intermittent or sustained elevated concentrations of SAA, CRP, or IL-6 (Supplementary Figure S7a,b).

\section{Low PDI}

When placental infection was followed by intermittent or sustained elevation of TNF- $\alpha$ or ICAM- 1 , the risk of a low PDI score was increased. These factors alone did not have any risk contribution (Supplementary Figure S8a,b).

\section{DISCUSSION}

We found considerable support for the hypothesis that two inflammatory stimuli result in an appreciably greater risk of white matter damage, in particular, for the three structural entities of ventriculomegaly, hypoechoic lesion, and microcephaly. Although ventriculomegaly reflects a diffuse loss of white matter, hypoechoic lesion reflects focal damage. We observed similar cytokine patterns for ventriculomegaly and hypoechoic lesion, suggesting similar causal pathways. Microcephaly at age 2 years can be a consequence of each of these early brain abnormalities (24).

Animal models (25-32) support a multi-hit hypothesis for white matter damage. Most of these studies involved hypoxiaischemia and inflammatory stimuli as exposures. A two-hit model for brain damage in human newborns showed that fetal growth restriction followed by postnatal systemic inflammation was associated with an increased risk of very low Bayley MDI (33). The risk of ventriculomegaly is increased when acute placental inflammation is superimposed on chronic placental inflammation, and when acute placental inflammation is followed by postnatal inflammation-initiating illnesses (34). Moreover, the risk of MRI-defined progressive white matter damage is increased when the preterm newborn has experienced multiple postnatal inflammatory disorders (35).

To study the two-hit model, we chose prenatal inflammation and postnatal systemic inflammation as our two hits. These two hits are separate chronologically. We also consider them separate based on findings that show more prominent inflammatory response documented on blood collected on postnatal days 7 and 14 . This would be beyond the period that the direct effects of placental inflammation would be seen, assuming the short half-life of the inflammatory proteins $(14,36,37,38)$. However, there is an association between the histologic inflammation of placenta and postnatal inflammation (39). They may represent two different time points of one long sustained inflammation, or exposure to inflammation before birth may leave the infant more susceptible to postnatal insults and predispose to systemic inflammation.

\section{Inflammation}

Intermittent or sustained systemic inflammation of the preterm newborn is characterized by elevated concentrations 
of proinflammatory cytokines in the systemic circulation at multiple time points (6). It is not known whether elevated concentrations of these cytokines are in response to multiple stimuli or to one initial stimulus and diminished resolution of the response.

Preterm infants are exposed to multiple postnatal insults that are associated with systemic inflammation, such as mechanical ventilation, bacteremia, and intestinal disease $(40,41)$, which might be in the causal chain between perinatal systemic inflammation and limitations identified at age 2 years. Consequently, we have not adjusted for these possible intervening variables. Previous results from this study sample demonstrated different protein profiles between infants born to mothers with histologic findings of inflammation in their placenta and infants born for maternal or fetal indications, such as preeclampsia and growth restriction (39). Therefore, the neonatal inflammatory response might be a continuation of fetal inflammatory response. Bacterial colonization of the very preterm placenta has been shown to be associated with distinct microorganism-specific inflammatory protein profiles in the newborn blood specimens (42). This also suggests that postnatal inflammation might have been triggered by events that started before birth.

\section{Sensitization}

The two-hit hypothesis may be considered an example of the first hit functioning as a sensitizer for the second hit. Placental inflammation might sensitize the newborn to respond more vigorously, and for a longer period of time, to a stimulus that would not otherwise have evoked such an intense response. Similarly, at the level of the brain, a subthreshold first (prenatal) stimulus may sensitize the brain tissue so that a second (postnatal) stimulus produces an increased response or damage compared with the response that would have occurred without the sensitizer (26-28,31).

\section{Strengths and Limitations}

Our study has several strengths. First, we included a large number of infants, making it unlikely that we have missed important associations because of the lack of statistical power. Second, we selected infants based on gestational age, not birth weight, to minimize confounding because of factors related to fetal growth restriction (43). Third, we collected all of our data prospectively. Fourth, attrition in the first 2 years was modest. Fifth, our protein data are of high quality $(13,14,16)$ and have high content validity $(38,39,42-44)$.

The limitations of our study are those of all observational studies. We are unable to distinguish between causation and association as explanations for what we found. Also, we evaluated the two-hit hypothesis and not the multi-hit hypothesis, which might be applicable if three or more hits are needed to appreciably increase the risk of any outcome of interest.

\section{CONCLUSION}

Our observation supports the hypothesis that placental inflammation followed by postnatal systemic inflammation predisposes to indicators of white matter damage on cranial ultrasound, cerebral palsy, low developmental indices, and microcephaly. This finding points to the complexity of the mechanisms leading to white matter damage, and of the importance of models to predict this outcome and its consequences. Because intermittent or sustained systemic inflammation observed in an extremely preterm newborn is a complex phenomenon that includes many proteins from multiple functional categories affecting multiple systems, we are reluctant to see the proteins we measured as anything more than indicators of a very broad set of interlinking processes. We believe that the individual cytokines are just messengers of this inflammation rather than targets in the pathogenesis of brain damage. We encourage further studies of the neonatal immune system. Identifying modulators of fetal and/or neonatal inflammation is crucial for the development of preventive and therapeutic interventions to reduce the preterm newborn's risk of white matter damage and adverse neurodevelopmental outcome.

\section{SUPPLEMENTARY MATERIAL}

Supplementary material is linked to the online version of the paper at http://www.nature.com/pr

\section{STATEMENT OF FINANCIAL SUPPORT}

This study was supported by The National Institute of Neurological Disorders and Stroke (5U01NS040069-05; 2R01NS040069-06A2) and the National Institute of Child Health and Human Development (5P30HD018655-28).

\section{REFERENCES}

1. Vohr BR, Wright LL, Poole WK, McDonald SA. Neurodevelopmental outcomes of extremely low birth weight infants <32 weeks' gestation between 1993 and 1998. Pediatrics 2005;116:635.

2. Kuban KC, Joseph RM, O'Shea TM, et al. Girls and boys born before 28 weeks gestation: risks of cognitive, behavioral, and neurologic outcomes at age 10 years. J Pediatr 2016;173:69.

3. Back SA, Riddle A, McClure MM. Maturation-dependent vulnerability of perinatal white matter in premature birth. Stroke 2007;38:724-30.

4. Dean JM, Shi Z, Fleiss B, et al. A critical review of models of perinatal infection. Dev Neurosci 2015;37:289-304.

5. Hagberg H, Mallard C, Ferriero DM, et al. The role of inflammation in perinatal brain injury. Nat Rev Neurol 2015;11:192-208.

6. Dammann $\mathrm{O}$, Leviton A. Intermittent or sustained systemic inflammation and the preterm brain. Pediatr Res 2014;75:376-80.

7. Kaukola T, Herva R, Perhomaa M, et al. Population cohort associating chorioamnionitis, cord inflammatory cytokines and neurologic outcome in very preterm, extremely low birth weight infants. Pediatr Res 2006;59: 478-83.

8. McElrath TF, Allred EN, Boggess KA, et al. Maternal antenatal complications and the risk of neonatal cerebral white matter damage and later cerebral palsy in children born at an extremely low gestational age. Am J Epidemiol 2009;170:819-28.

9. Denzler A, Burkhardt T, Natalucci G, Zimmermann R. Latency after preterm prelabor rupture of the membranes: increased risk for periventricular leukomalacia. J Pregn 2014;2014:874984.

10. Hatfield T, Wing DA, Buss C, Head K, Muftuler LT, Davis EP. Magnetic resonance imaging demonstrates long-term changes in brain structure in 


\section{Articles $\mid$ Yanni et al.}

children born preterm and exposed to chorioamnionitis. Am J Obstet Gynecol 2011;205384:e1-8.

11. Pogribna U, Yu X, Burson K, et al. Perinatal clinical antecedents of white matter microstructural abnormalities on diffusion tensor imaging in extremely preterm infants. PLoS ONE 2013;8:e72974.

12. O'Shea TM, Shah B, Allred EN, et al. Inflammation-initiating illnesses, inflammation-related proteins, and cognitive impairment in extremely preterm infants. Brain Behav Immun 2013;29:104-2.

13. Leviton A, Kuban K, O'Shea TM, et al. The relationship between early concentrations of 25 blood proteins and cerebral white matter injury in preterm newborns: the ELGAN study. J Pediatr 2011;158:897-903.

14. O'Shea TM, Allred EN, Kuban KC, et al. Elevated concentrations of inflammation-related proteins in postnatal blood predict severe developmental delay at two years in extremely premature infants. J Pediatr. 2012;160:395-401.

15. Leviton A, Kuban KC, Allred EN, Fichorova RN, O'Shea TM, Paneth N. Early postnatal blood concentrations of inflammation-related proteins and microcephaly two years later in infants born before the 28th postmenstrual week. Early Hum Dev 2011;87:325-30.

16. O'Shea TM, Allred EN, Dammann O, et al. The ELGAN study of the brain and related disorders in extremely low gestational age newborns. Early Hum Dev 2009;85:719-25.

17. Driscoll SG, Langston C. College of American Pathologists Conference XIX on the examination of the placenta: report of the working group on methods for placental examination. Arch Pathol Lab Med 1991;115:704-8.

18. Hecht JL, Onderdonk A, Delaney M, et al. Characterization of chorioamnionitis in 2nd-trimester C-section placentas and correlation with microorganism recovery from subamniotic tissues. Pediatr Dev Pathol 2008;11:15-22.

19. Hecht JL, Alred EN, Kliman HJ, et al. Histological characteristics of singleton placentas delivered before the 28th week of gestation. Pathology 2008;40:372-6.

20. Kuban KC, Allred EN, O'Shea TM, et al. ELGAN study investigators. Cranial ultrasound lesions in the NICU predict cerebral palsy at age 2 years in children born at extremely low gestational age. J Child Neurol 2009;24:63-72.

21. Kuban K, Adler I, Allred EN, et al. Observer variability assessing US scans of the preterm brain: the ELGAN study. Pediatr Radiol 2007;37:1201-8.

22. Bayley N. Bayley Scales of Infant Development-II. San Antonio, TX: Psychological Corporation, 1993.

23. Roberts G, Anderson PJ, Doyle LW. The stability of the diagnosis of developmental disability between ages 2 and 8 in a geographic cohort of very preterm children born in 1997. Arch Dis Child 2010;95:786-90.

24. Krishnamoorthy KS, Kuban KC, O'Shea TM, Westra SJ, Allred EN, Leviton A. Early cranial ultrasound lesions predict microcephaly at age 2 years in preterm infants. J Child Neurol 2011;26:188-94.

25. Dommergues MA, Patkai J, Renauld JC, Evrard P, Gressens P. Proinflammatory cytokines and interleukin-9 exacerbate excitotoxic lesions of the newborn murine neopallium. Ann Neurol 2000;47:54-63.

26. Eklind S, Mallard C, Leverin AL, et al. Bacterial endotoxin sensitizes the immature brain to hypoxic-ischaemic injury. Eur J Neurosci 2001;13: $1101-6$.

27. Eklind S, Mallard C, Arvidsson P, Hagberg H. Lipopolysaccharide induces both a primary and a secondary phase of sensitization in the developing rat brain. Pediatr Res 2005;58:112-6.
28. Bilbo SD, Biedenkapp JC, Der-Avakian A, Watkins LR, Rudy JW, Maier SF. Neonatal infection-induced memory impairment after lipopolysaccharide in adulthood is prevented via caspase-1 inhibition. J Neurosci. 2005;25:8000-9.

29. Bilbo SD, Levkoff LH, Mahoney JH, Watkins LR, Rudy JW, Maier SF. Neonatal infection induces memory impairments following an immune challenge in adulthood. Behav Neurosci 2005;119 p 293-301.

30. Wang X, Hagberg H, Nie C, Zhu C, Ikeda T, Mallard C. Dual role of intrauterine immune challenge on neonatal and adult brain vulnerability to hypoxia-ischemia. J Neuropathol Exp Neurol 2007;66:552-61.

31. Kaindl AM, Favrais G, Gressens P. Molecular mechanisms involved in injury to the preterm brain. J Child Neurol 2009;24:1112-8.

32. Williamson LL, Sholar PW, Mistry RS, Smith SH, Bilbo SD. Microglia and memory: modulation by early-life infection. J Neurosci 2011;31: 15511-21.

33. Leviton A, Fichorova RN, O'Shea TM, Kuban K, Paneth N, Dammann O. Allred E for the ELGAN Study Investigators. Two-hit model of brain damage in the very preterm newborn: small for gestational age and postnatal systemic inflammation. Pediatr Res 2013;73:362-70.

34. Korzeniewski SJ, Romero R, Cortez J, et al. A 'multi-hit' model of neonatal white matter injury: cumulative contributions of chronic placental inflammation, acute fetal inflammation and postnatal inflammatory events. J Perinat Med 2014;42:731-43.

35. Glass HC, Bonifacio SL, Chau V, et al. Recurrent postnatal infections are associated with progressive white matter injury in premature infants. Pediatrics 2008;122:299-305.

36. Patel S, Dammann O, Martin CR, Allred EN. Leviton A for the ELGAN Study Investigators. Presumed and definite bacteremia in extremely low gestational age newborns. Acta Paediatr 2011;100:36-41.

37. Leviton A, Hecht JL, Allred EN, Yamamoto H, Fichorova RN, Dammann O. Persistence after birth of systemic inflammation associated with umbilical cord inflammation. J Reprod Immunol 2011;90: 235-43.

38. Leviton A, Fichorova R, Yamamoto $\mathrm{Y}$, et al. Inflammation-related proteins in the blood of extremely low gestational age newborns. The contribution of inflammation to the appearance of developmental regulation. Cytokine 2011;53:66-73.

39. McElrath TF, Fichorova RN, Allred EN, et al. Blood protein profiles of infants born before 28th weeks differ by pregnancy complication. Am J Obstet Gynecol 2011;204:418.

40. Bose C, Laughon M, Allred EN, et al. Systemic inflammation associated with mechanical ventilation among extremely preterm infants. Cytokine 2013;61:315-22.

41. Martin CR, Bellomy M, Allred EN, Fichorova RN, Leviton A. Systemic inflammation associated with severe intestinal injury in extremely low gestational age newborns. Fetal Pediatr Pathol 2013;32:222-34.

42. Fichorova RN, Onderdonk AB, Yamamoto H, et al. Maternal microbespecific modulation of inflammatory response in extremely lowgestational-age newborns. mBio 2011;2:00280-00210.

43. Arnold CC, Kramer MS, Hobbs CA, McLean FH, Usher RH. Very low birth weight: a problematic cohort for epidemiologic studies of very small or immature neonates. Am J Epidemiol 1991;134:604-13.

44. Hecht JL, Fichorova RN, Tang VF, et al. The relationship between neonatal blood protein profiles and placenta histologic characteristics in ELGANs. Pediatr Res 2011;69:68-73. 